\title{
REGISTROS DA ATIVIDADE SOLAR NOS ANÉIS DE CRESCIMENTO DE ÁRVORES EM SÃO FRANCISCO DE PAULA - RS (BRASIL)
}

\author{
N. R. Rigozo ${ }^{1}$ \& D. J. R. Nordemann ${ }^{2}$
}

$\mathrm{O}$ aspecto mais conhecido da variabilidade solar é o ciclo de 11 anos, observado no número de manchas solares. No entanto, este ciclo tem sido observado em outros registros, como os das atividades nucleares dos cosmonuclídeos ${ }^{14} \mathrm{Ce}{ }^{10} \mathrm{Be}$ em testemunhos de gelo das calotas polares. Cronologias dos índices das espessuras dos anéis de crescimento das árvores, recentemente, começaram a ser usadas como possíveis registros das variações do ciclo solar no passado. Em diferentes locais no mundo foi observado uma influência do ciclo solar de 11 anos nos anéis de crescimento de árvores. Entretanto em outros locais não foi possível encontrar vestígios dessa influência solar nas árvores. Isto nos levou a fazer um estudo das possíveis influências da atividade solar nos anéis de crescimento de árvores da América do Sul. A determinação das espessuras de anéis é feita através de uma metodologia ótica e computacional. Este trabalho mostra os resultados obtidos para um destes locais, que é São Francisco de Paula, Brasil. Uma cronologia média de 65 anos foi determinada para as espessuras dos anéis de crescimento de árvores, neste lugar. Para a procura das periodicidades, relacionadas com os fenômenos de origem solar, foi usado o método de análise da regressão iterativa. Nós encontramos periodicidades de 20,7, 12,8 e 5,6 anos relacionadas aos ciclos solares de 22 anos (Ciclo de Hale), 11 anos (ciclo de Schwabe) e o segundo harmônico do ciclo de 11 anos. Também foi encontrado um período de 8,6 anos, o qual é devido ao desbaste feito na floresta.

Palavras-chave: Anéis de árvores; Atividade solar; Análise espectral; Regressão iterativa; Análise de imagem.

RECORD OF SOLAR ACTIVITY IN TREE GROWTH-RINGS FROM SÃO FRANCISCO DE PAULA - RS (BRAZIL)-The most important aspect of the solar variability is the 11 year-old cycle, observed in sunspot number variations. This cycle has already been observed in terrestrial records, such as the nuclear activity variations of cosmogenic ${ }^{14} \mathrm{C}$ from tree rings and ${ }^{10} \mathrm{Be}$ in ice sample of polar caps, by sophisticated and high cost methods. By more simple methods, chronologies of tree ring width index recently began to be used as possible record of the solar activity variations in the past. In different locations of the world an influence of the 11 year-old solar cycle was observed in tree growth rings. However, in some other places it was not possible to find the influence of solar activity variations in trees. This led us to perform a study of the possible influence of the solar activity in tree rings from trees grown in South America. The tree rings width determination is made using an optical and computational methodology. This work shows the results obtained at one of the locations studied, at San Francisco of Paula, Brazil. A 65 year-old mean chronology was determined for the tree ring width at this place. For the periodicity search, related to the phenomena of solar origin, the iterative regression analysis method was used. Periods were found at 20.7, 12.8 and 5.6 years related to the cycles 22 year solar (Hale Cycle), 11 year (Schwabe cycle) and the second harmonic of the 11 year cycle. It was also found a 8.6 year period, which is due to periodical thinning down for forest management and preservation.

Key words: Tree ring; Solar activity; Spectral Analysis; Iterative regression; Image analysis.

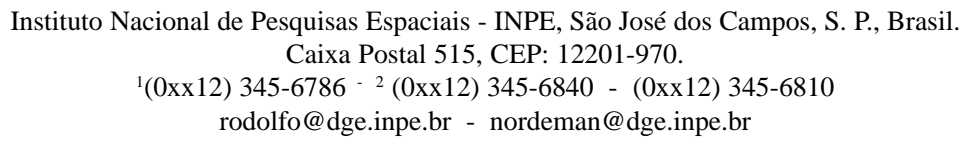




\section{INTRODUÇÃO}

$\mathrm{O}$ aspecto mais conhecido da variabilidade solar é o ciclo de 11 anos, descoberto por A. N. Schwabe em 1843. Este ciclo se deve às inversões do campo magnético solar, que provocam, periodicamente, uma maior emissão de partículas e radiação, na qual ocorre o aumentando da atividade solar.

Longas cronologias das variações da atividade solar têm sido reconstruídas a partir de uma variedade de fontes. Schrove (1955) usou registros históricos das observações das manchas solares e da atividade auroral para estimar as magnitudes e os anos em que ocorreram o máximo do número de manchas solares, desde o ano 300 d.C. Separadamente ao ciclo médio de 11 anos, ele identificou um ciclo de 78 anos (chamado ciclo de Gleissberg) na atividade das manchas solares, e um ciclo de 200 anos para a atividade auroral. Mudanças nos níveis de ${ }^{14} \mathrm{C}$ atmosférico, também têm sido relacionadas as variações da atividade solar (Stuiver \& Quay, 1980).

Cronologias dos índices das espessuras dos anéis de crescimento das árvores também têm sido usados como registros das variações do ciclo solar no passado. Os modelos das variações das espessuras dos anéis geralmente indicam a presença de longos e curtos períodos na fase de crescimento. Em alguns casos, um significante ciclo de 11 anos é evidente, com um pequeno atraso de tempo, com respeito ao ciclo solar (Douglass, 1928; Mori, 1981).

Este trabalho apresenta alguns resultados da procura de periodicidades, associadas a atividade solar, em séries temporais dos anéis de crescimento de árvores, para a região sul do Brasil. As medidas das espessuras dos anéis de crescimento foram feitas por uma metodologia de baixo custo, sendo ela inteiramente computacional, pela qual se identifica e mede as espessuras dos anéis de crescimento de árvores. Isso é feito através de imagens digitalizadas, por um digitalizador de mesa, transformando-as em séries temporais. Para isso desenvolveu-se um programa computacional interativo, no ambiente do Interactive Data Language (IDL). As análises das séries temporais são feitas pelo método da regressão iterativa, devido a sua precisão e a determinação dos desvios padrões sobre os resultados.

\section{DADOS}

Foram obtidas 12 amostras de árvores em forma de disco da espécie nativa Araucaria angustifolia e uma amostra da espécie não nativa Pinus taeda, para investigar a possibilidade da atividade solar induzir periodicidades nos anéis de crescimento das árvores de São Francisco de Paula - RS (lat. 29 25' S, lon. 50 24' O, alt. 930m). As séries das espessuras dos anéis de crescimento foram obtidas através das imagens digitalizadas dos seus anéis de crescimento, de cada uma das amostras, por uma metodologia digital, denominada de Tratamento Interativo de Imagens dos Anéis de Árvores (TIIAA) (Rigozo \& Nordemann, 1999). As medidas dos erros, na determinação das espessuras dos anéis de crescimento, são estimadas em torno de 1 a 2 pixel/anel. A maior cronologia determinada foi de 66 anos, que se estende de 1931 a 1996. A Fig. 1 apresenta as séries temporais das espessuras dos anéis de crescimento de árvores, para 13 amostras coletadas em São Francisco de Paula - RS.

Antes de efetuar as análises espectrais das séries temporais, removem-se as tendências de longos períodos contidas nos anéis de crescimentos, de cada amostra. Na remoção dessas tendências, utilizou-se a subtração de uma função gaussiana nas 13 séries. Para a obtenção da série temporal representativa do local, efetuou-se a média entre as 13 séries temporais, já sem as suas respectivas tendências. Seguindo a idéia de Kurths et al. (1993), fez-se ainda uma subtração de uma função polinomial de terceira ordem na série representativa do local (Fig. 2). Isso é feito porque nem sempre se consegue escolher a melhor curva de ajuste que represente as tendências de crescimento das árvores, o que torna necessário usar mais de uma curva de ajuste, para eliminar resíduos de longos períodos, que ainda se encontram na série temporal.

\section{ANÁLISE ESPECTRAL}

Nesse estudo, utilizou-se o método de análise espectral da regressão iterativa, na procura das periodicidades contidas nas séries dos anéis de crescimento de árvores. O método de Análise por Regressão Iterativa de Séries Temporais (ARIST) utiliza uma simples função senoídal com três parâmetros desconhecidos, $\mathbf{a}_{\mathbf{0}}=$ amplitude, $\mathbf{a}_{\mathbf{1}}=$ freqüência angular e $\mathbf{a}_{2}=$ fase da função (Wolberg, 1967; Nordemann, 1992; Rigozo, 1994). O ponto inicial do método é a definição da chamada função condicional, que é:

$$
\mathrm{F}=\mathrm{Y}-a_{0} \sin \left(a_{1} t+a_{2}\right),
$$

onde $\mathbf{Y}$ é o sinal medido, $\mathbf{t}$ é o tempo e $\mathbf{a}_{\mathbf{0}}, \mathbf{a}_{\mathbf{1}}, \mathbf{a}_{\mathbf{2}}$ são os três parâmetros desconhecidos, que serão procurados por iterações através da minimização do quadrado desta função $\mathrm{F}$. O número máximo de iterações utilizado na 


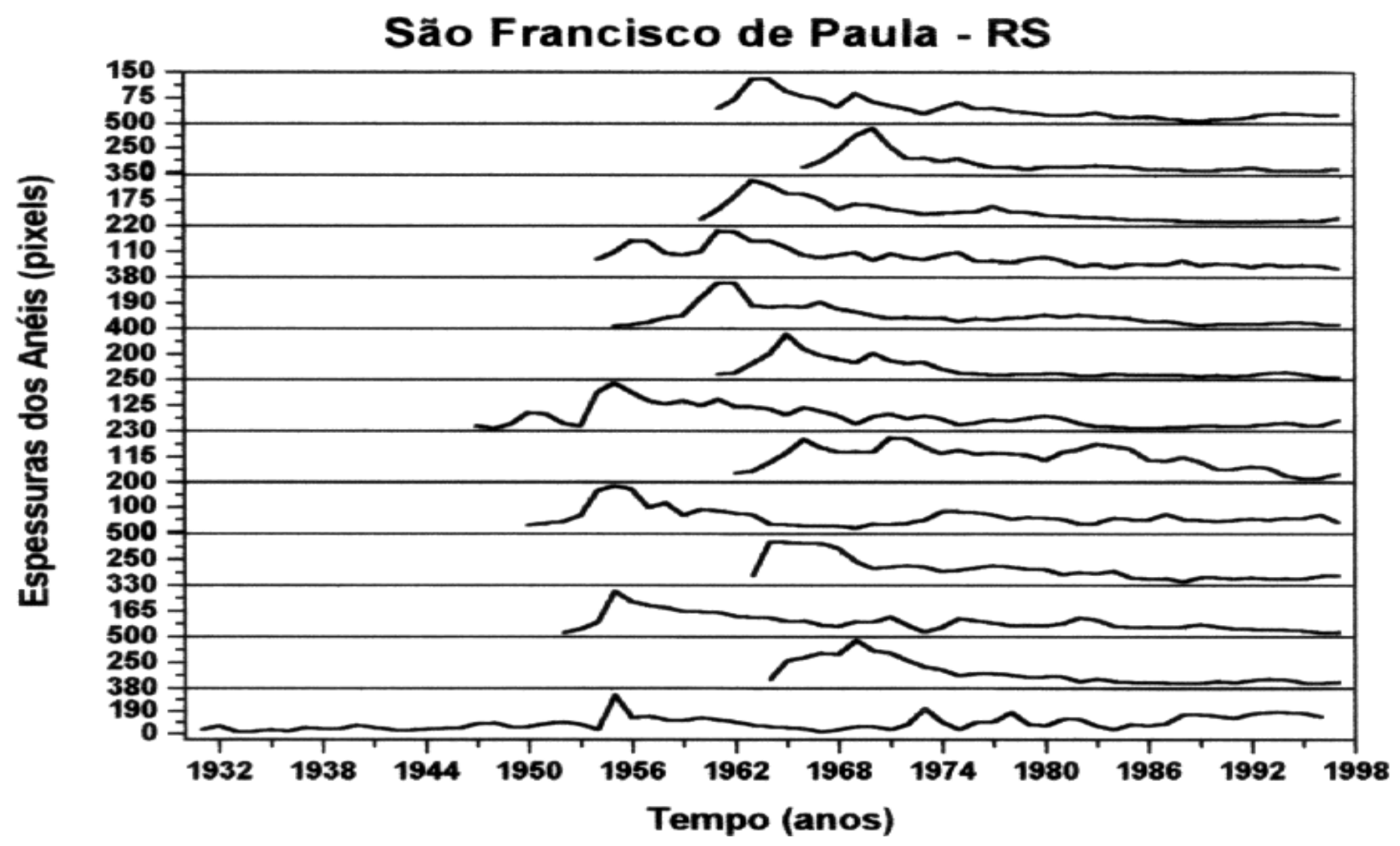

Figura 1- Séries temporais das espessuras dos anéis de crescimento de árvores, para 13 amostras coletadas em São Francisco de Paula-RS.

Figure 1 - Tree growth-ring width time series for 13 samples from São Francisco de Paula-RS.

Cronologia dos Anéis de Crescimento de Árvores

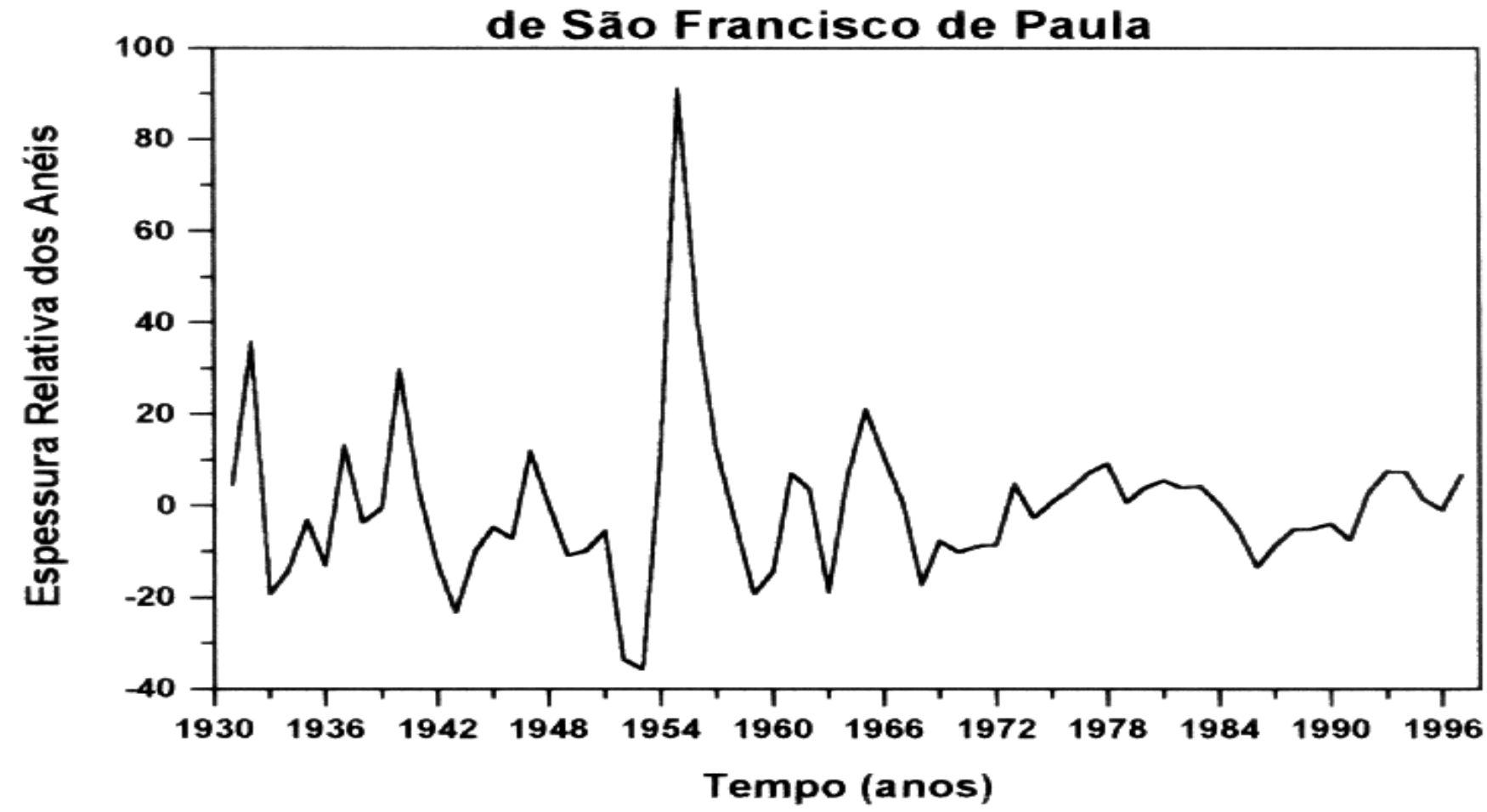

Figura 2 - Série temporal média das espessuras dos anéis de crescimento de árvores, para São Francisco de Paula-RS.

Figure 2 - Tree growth-ring width mean time series from São Francisco de Paula-RS. 


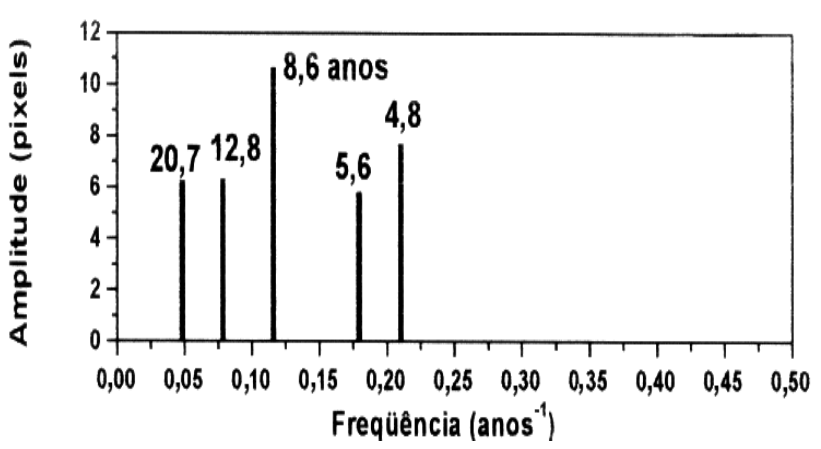

Figura 3- Espectro de amplitude determinado pelo método da regressão iterativa.

Figure 3-Amplitude spectrum determined by the iterative regression method..

determinação dos três parâmetros foi de 100 (Rigozo \& Nordemann, 1998). A vantagem deste método é que ele fornece o desvio padrão de cada um dos três parâmetros determinados. Isto permite uma seleção das amplitudes mais importantes, que apresentam maiores valores na relação amplitude/desvio.

\section{RESULTADOS}

Após a eliminação das tendências de longos períodos, na série temporal média das espessuras dos anéis de crescimento de árvores, a série obtida é representativa das variações de médio e curto período neste caso no município de São Francisco de Paula - RS. Efetuam-se as análises espectrais dessa série. A Fig. 3 apresenta o espectro de amplitude em função da frequiência, determinado pelo método da regressão iterativa. A Tab. 1 apresenta as periodicidades determinadas pelo mesmo método. Também são apresentados os desvios padrões para cada período e amplitude encontrada.

O nosso interesse é de observar periodicidades pertencentes aos ciclos da atividade solar nas espessuras dos anéis de crescimento de árvores. Assim, encontraram-se três períodos que podem ser característicos da atividade solar. O período de 12,8 anos pode representar o ciclo solar de 11 anos, no qual o Sol sofre uma inversão dos seus pólos magnéticos, o que provoca uma maior emissão de partículas e radiação, acarretando numa maior atividade solar (Stuiver \& Quay, 1980; Eddy 1980). Também se observou um período de 20,7 anos, que pode ser associado ao ciclo solar de Hale de 22 anos. Este comportamento cíclico de 22 anos é representado pela reversão dos pólos magnético do Sol, ou seja o campo magnético volta ao seu sentido original (Mitchell et al., 1979). Ainda se encontrou um período de 5,6 anos. Existem indicações recentes, a partir do número de manchas solares e de

\begin{tabular}{|c|c|c|c|}
\hline $\begin{array}{c}\text { Período } \\
\text { (anos) }\end{array}$ & $\begin{array}{c}\text { Desvio } \\
\text { (anos) }\end{array}$ & $\begin{array}{c}\text { Amplitude } \\
\text { (pixels) }\end{array}$ & $\begin{array}{c}\text { Desvio } \\
\text { (pixels) }\end{array}$ \\
\hline 20,8 & 1,6 & 06,3 & 2,9 \\
\hline 12,8 & 0,6 & 06,3 & 3,0 \\
\hline 08,6 & 0,2 & 10,7 & 2,8 \\
\hline 05,6 & 0,1 & 05,8 & 2,9 \\
\hline 04,8 & 0,1 & 07,7 & 2,9 \\
\hline
\end{tabular}

Tabela 1 - Os períodos e amplitudes determinados pelo método da regressão iterativa, juntamente com seus respectivos desvios padrões.

Table 1 - Periods and amplitudes determined by spectral analysis, and their respective standard deviations.

dados climáticos, sobre um período em torno de 5,5 anos da atividade solar (Vitinsky et al., 1986), conhecido como segundo harmônico do ciclo solar de 11 anos (Damon \& Jirikowic, 1992).

Observa-se que o sinal mais dominante se encontra em 8,6 anos. Esse período se deve principalmente ao desbaste seletivo, efetuado a cada 8 anos pelos engenheiros florestais. Esses desbastes consistem em derrubar árvores velhas ou que apresentem algum tipo de anomalia, para que as árvores mais novas e em boas condições de saúde consigam se desenvolverem melhor. Consequentemente isso provoca um maior crescimento nessas árvores, pois elimina-se assim a concorrência por água, nutrientes e luz solar.

$\mathrm{Na}$ tentativa de identificar melhor os sinais de freqüências, contidos nas 13 séries temporais das espessuras dos anéis de crescimento de árvores, fez-se um histograma das freqüências, obtidas em cada uma das séries. O histograma (Fig. 4) mostra os resultados obtidos. Os pontos que aparecem nos gráficos correspondem à frequiência (ou período) central da banda espectral, dominante em cada espectro de amplitude.

O histograma apresenta as periodicidades mais características nas 13 amostras, dos anéis de crescimento de árvores. Observa-se que as periodicidades dominantes são devidas a uma possível influência da atividade solar, para os períodos de 21,5 (com uma ocorrência de 7 vezes) e 12,9 anos (com uma ocorrência de 5 vezes) (Fig. 4), que caracterizam os ciclos solares de Hale e o ciclo de Schwabe, no crescimento dos anéis.

Os resultados do histograma confirmam as principais periodicidades obtidas, através da análise da série temporal média das espessuras dos anéis de árvores. Isso indica que não houve perda ou introdução de algum sinal de freqüência, no procedimento de 


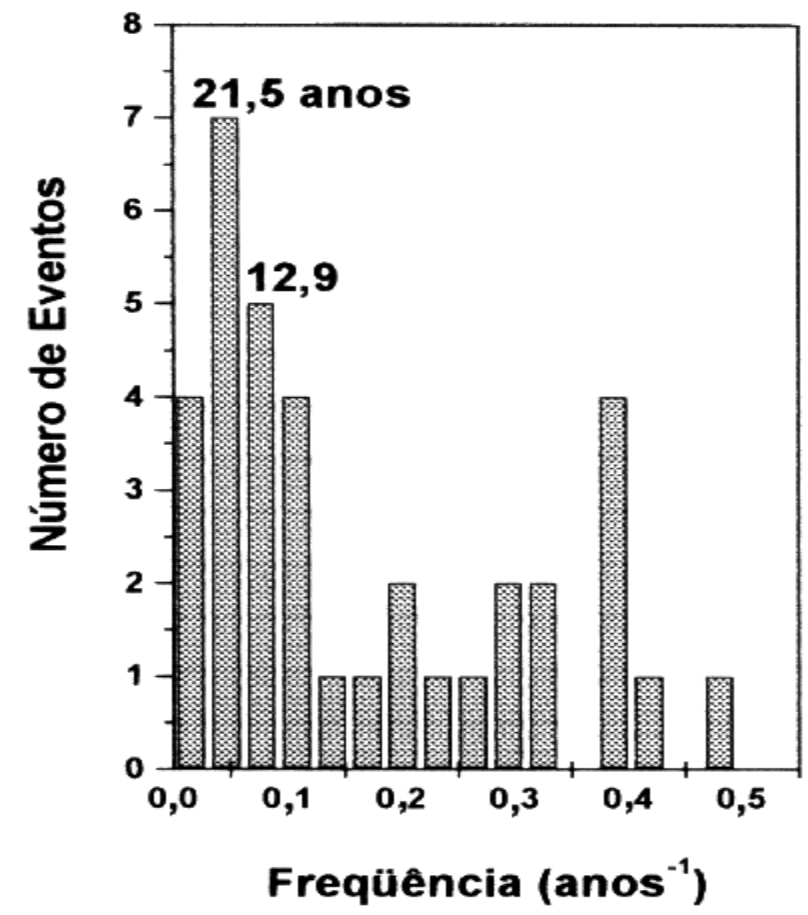

Figura 4 - Histograma dos resultados obtidos pelo método da regressão iterativa das 13 amostras dos anéis de crescimento.

Figure 4 - Histogram of the number of events as a function of frequency for the 13 tree growth-ring samples.

obtenção da série temporal representativa de São Francisco de Paula, através da média entre as séries das espessuras dos anéis (sem as suas respectivas tendências de crescimento).

\section{DISCUSSÕES E CONCLUSÕES}

Os fatores climáticos e as condições ambientais locais representam uma maior influência no crescimento dos anéis de árvores. Espera-se que qualquer influência solar, a partir das variações de sua irradiação, deva ser menor. No entanto, há confirmações de que variações nas espessuras dos anéis de crescimento são atribuídas, em parte, à atividade solar. Essas confirmações são baseadas em estudos feitos em outras séries de anéis de crescimento, obtidas a partir de outras espécies de árvores em locais com contrastes no clima. Assim, Murphy (1990 e 1991) observou periodicidades entre 9,3 a 13,3 anos em anéis de crescimento de árvores da Austrália, e períodos de 11,1 e 13,6 anos, nos anéis de crescimento de árvores de Taiwan. Ele atribuiu estas periodicidades às influências do ciclo solar de 11 anos. Esses mesmos períodos, referentes ao ciclo solar de 11 anos, foram encontrados por nós em anéis de crescimento de árvores em São Francisco de Paula. Nos mesmos estudos dos anéis de crescimento de árvores da Austrália e Taiwan,
Murphy também encontrou períodos em torno de 20 a 25 anos, pertencentes ao ciclo de Hale. Nós, também, observamos uma possível influência do ciclo solar de 22 anos, nas árvores de São Francisco de Paula. O histograma apresentou uma maior ocorrência para o ciclo de Hale. O passado longínquo, também, evidencia uma forte influência da atividade solar sobre o crescimento das árvores. Kurths et al. (1993) estudando amostras fossilizadas dos troncos de árvores, de aproximadamente 15 a 20 milhões de anos atrás, encontradas na Alemanha, observaram períodos de 12 anos e 5,5 anos. Em são Francisco de Paula, para o passado mais recente, mostrou-se o segundo harmônico do ciclo solar de 11 anos, em 5,6 anos. Isso mostra uma semelhança nas variações solares do passado longínquo, com o passado recente.

Evidências convincentes e independentes das influências das periodicidades solar em séries temporais das espessuras dos anéis de árvores são obtidas pelas variações nos longos registros do ${ }^{14} \mathrm{C}$ (Stuiver \& Quay, 1980). Kocharov et al. (1995) estudando os conteúdos do $\mathrm{D}^{14} \mathrm{C}$, na celulose de anéis de crescimento de árvores de Bashkiria - Rússia, encontraram períodos de 21,4 anos, 9,4-9,6 anos e 5,5 anos, com o método de análise de Fourier; e períodos entre 21,3 anos, 9,7 a 13,5 anos e 5,5 anos, com o método de análise da máxima entropia, que eles atribuíram às influências da atividade solar. Recentemente, Damon et al. (1998) estudando uma série temporal do $\mathrm{D}^{14} \mathrm{C}$ em anéis de crescimento de árvores, para o intervalo de tempo entre 1065 a 1250, encontraram períodos de 24,3 anos, 9,8-13,9 anos e 5,5 anos. Eles atribuíram esses períodos ao ciclo solar de Hale, ciclo solar de 11 anos e ao segundo harmônico do ciclo de 11 anos.

Em concordância com as periodicidades encontradas, nos registros do ${ }^{14} \mathrm{C}$ e nas séries das espessuras dos anéis de crescimento de árvores da Austrália, Taiwan e Alemanha, nós também observamos periodicidades pertencentes a uma possível influência da atividade solar. Os períodos encontrados foram 20,7, 12,8 e 5,6 anos, caracterizados pelos ciclos de Hale, ciclo de 11 anos e o segundo harmônico do ciclo de 11 anos, respectivamente. Também foi observado um período dominante, próximo dos 8 anos, devido aos desbaste seletivo.

Constatou-se ainda que a obtenção de uma série temporal média entre as séries de espessuras dos anéis (sem as suas respectivas tendências de crescimento), não introduziu ou eliminou qualquer sinal de freqüência que poderia prejudicar a observação dos sinais de origem solar. 


\section{AGRADECIMENTOS}

Ao colega Luís E. A. Vieira, pela ajuda na coleta das amostras de árvores em São Francisco de Paula. À Sra. Maria da Graça Hübbe pela sua ajuda na aquisição das amostras de árvore em São Francisco de Paula. Ao IBAMA pela doação das amostras de árvores. Ao Engenheiros Florestais do IBAMA, Artur José Soligo pela ajuda prestada em São Francisco de Paula. À Fundação de Amparo a Pesquisa do Estado de São Paulo (FAPESP) pelo auxilio de verbas, através do projeto número 97/14063-0 (Daniel J. R. Nordemann) e pela bolsa de pós-doutorado número 98/16094-2 (Nivaor R. Rigozo) e o CNPq pela bolsa de Produtividade em Pesquisa número 300031/94-7 (Daniel J. R. Nordemann).

\section{REFERÊNCIAS}

DAMON, P. E. \& JIRIKOWIC, J. L. - 1992 - The sun as a low-frequency harmonic oscillator. Radiocarbon, 34(2):199-205.

DAMON, P. E., EASTONE, C. J., HUGHES, M. K., KALIN, R. M., LONG, A. \& PERISTYKH, A. N. 1998 - Secular variation of $\mathrm{D}^{14} \mathrm{C}$ during the medieval solar maximum: a progress report. Radiocarbon, 40(1):343-350.

DOUGLASS, A. E. - 1928 - Climatic cycles and tree growth. Vol. II, Carnegie Institute of Washington Publications, Washington, D.C.

EDDY, J. A. - 1980 - The historical record of solar activity. Proceedings of the conference on the ancient sun: fossil record in the earth, moon and meteorites. 119-134.

KOCHAROV, G. E., OSTRYAKOV, V. M., PERISTYKH, A. N. \& VASIL'EV, V. A. - 1995 Radiocarbon content variations and Maunder minimum of solar activity. Solar Physics, 159:381-391.

KURTHS, J., SPIERING, CH., MÜLLER-STOLL, W. \& STRIEGLER, U. - 1993 - Search for solar periodicities in Miocene tree ring widths. Terra Nova, 5: 359-363.

MITCHELL, S. M. JR., STOCKTON, C. W. \& MEKO, D. M. - 1979 - Evidence of a 22-year rhythm of drought in the Western United States related to the Hale solar cycle since the $17^{\text {th }}$ century. Solar Terrestrial Influences on Weather and Climate, Reidel, Dordrecht, Holland, $125-143$.

MORI, Y. - 1981 - Evidence of an 11-year periodicity in tree-ring series from Formosa related to the sunspot cycle. Journal Climatology, 1:345-353.

MURPHY, J. O. - 1990 - Australian tree ring chronologies a proxy data for solar variability. Proceedings ASA, 8(3): 292-297.

MURPHY, J. O. - 1991 - The downturn in solar activity during solar cycles 5 and 6. Proceedings ASA, 9(2):330331.

NORDEMANN, D. J. R. - 1992 - Search for periodicities in geophysical time series by iterative regression analysis in C. São José dos Campos, INPE, 20pp. (INPE5420-PRE/1762).

RIGOZO, N. R. - 1994 - Análise por regressão iterativa de periodicidades nas variações dos cosmonuclídeos atmosféricos Be-10 e C-14. São José dos Campos, 79pp. (INPE-5547-TDI/533). Dissertação (Mestrado em Geofísica Espacial) - Instituto Nacional de Pesquisas Espaciais.

RIGOZO, N. R. \& NORDEMANN, D. J. R. - 1998 Análise por regressão iterativa de periodicidades em séries temporais de registros geofísicos. Revista Brasileira de Geofísica 16 (2/3): 149-157.

RIGOZO, N. R. \& NORDEMANN, D. J. R. - 1999 - Solar activity records in tree-rings. Revista Geofísica Instituto Panamericano de Geografía e História (México), 50:163-170.

SCHROVE, D. J. - 1955 - Journal of Geophysical Research, 60:127.

STUIVER, M. \& QUAY, P. D. - 1980 - Changes in atmospheric carbon-14 attributed to a variable Sun. Science, 207(4426):11-19.

VITINSKY, JU. I., KOPETZKI, M. \& KUKLIN G. B. 1986 - Statistics of spot-generating activity of the Sun. Nauka, Moscow.

WOLBERG, J. R. - 1967 - Prediction analysis. Princeton, D. Van Nostrand Company, 293pp.

Manuscript submitted June 24, 1999 Revised version accepted February 10, 2000

\section{NOTE ABOUT THE AUTHORS}

\section{Daniel Jean Roger Nordemann}

Formou-se em Engenharia Física pela École Supérieure de Physique et Chimie Industrielles (ESPCI) de Paris, França em 1957. Bacharel em Física Matemática em 1963 e Doutor em Ciências Físicas em 1966 pela Universidade de Paris, França. Trabalhou na França de 1957 a 1970 e de 1974 a 1979 no Commissariat à l'Énergie Atomique (CEA), onde desenvolveu trabalhos na área da Geofísica Nuclear. Trabalhou como perito da UNESCO, de 1970 a 1973, no Instituto de Física da Universidade Federal da Bahia (IF-UFBa). Desde 1979, trabalha no Instituto Nacional de Pesquisas Espaciais (INPE) nas áreas de Geofísica Espacial e Geofísica Nuclear. É autor de vários livros entre os quais "Elementos de Geocronologia Nuclear" (1972), "Introdução ao Mathematica for Windows" (1994) e "Problemas Neuro-metabólicos: Autismo e Candidíase" (1999).

\section{Nivaor Rodolfo Rigozo}

Graduou-se em Física pela Pontifícia Universidade Católica PUC (1991) de Porto Alegre. Mestre em Ciência Espacial pelo Instituto Nacional de Pesquisas Espaciais - INPE (1994) com uma dissertação intitulada "Análise por regressão iterativa de periodicidades nas variações dos cosmonuclídeos atmosféricos ${ }^{10} \mathrm{Be} e e^{14} \mathrm{C}^{\prime \prime}$. Doutorado em Geofísica Espacial pelo Instituto Nacional de Pesquisas Espaciais - INPE (1998) com tese intitulada "Registros da atividade solar e de outros fenômenos geofísicos em anéis de árvores". 


\title{
Record of Solar Activity in Tree Rings from São Francisco de Paula - RS (Brazil)
}

The most important aspect of the solar variability is the 11 year-old cycle, observed in sunspot number variations. This cycle has already been observed in terrestrial records, such as the nuclear activity variations of cosmogenic ${ }^{14} \mathrm{C}$ from tree rings and ${ }^{10} \mathrm{Be}$ in ice sample of polar caps, by sophisticated and high cost methods. By more simple methods, chronologies of tree ring width index recently began to be used as possible record of the solar activity variations in the past. In different locations in the world an influence of the 11 year-old solar cycle was observed in tree growth rings. However, in some other places it was not possible to find influence of that solar activity in trees. This took us to study the possible influence of the solar activity in tree rings from trees grown in South America.

The tree ring-width determination is made using an original low cost optical and computational method which identifies and measures growth ring thickness in polished slices prepared from the base of dead tree trunks. Digitized images of these slices are acquired by a flatbed scanner associated to a microcomputer and then processed in order to extract time series of mean radial ring widths from core to bark. An interactive routine was specially developed for this purpose within Interactive Data Language (IDL) environment. Time series are analyzed by an itera- tive regression method which provides the parameters (period, amplitude and phase) of the embedded periodical sine functions, with precision and their respective standard deviation values.

This work shows the results obtained at one of the locations studied, at San Francisco de Paula, Rio Grande do Sul State, Brazil. A 65 year-old mean chronology was determined for tree ring widths at this place. The conifer species used to search possible influence of solar variability were native Araucaria angustifolia and non-native Pinus taeda because of good contrast shown in their rings and possible adequate sensitivity to environment variations.

Periods were found at 20.7, 12.8 and 5.6 years related to the cycles 22 year solar (Hale Cycle), 11 year (Schwabe Cycle) and the second harmonic of the 11 year cycle. It was also found a 8.6 year period, which is due to periodical thinning down for forest management and preservation, and does not interfere with the results obtained on solar variability influence thanks to their clear respective period identifications. From these first results it may be concluded that the original method described is very convenient for such studies, mainly because of its precision and low cost. Preference also should be given to conifer native species, such as araucaria in Southern Brazil, in order to possibly analyze solar variation influences at longer time scales.

\section{UNIVERSIDADE FEDERAL DA BAHIA CENTRO DE PESQUISA EM GEOFÍSICA E GEOLOGIA} CPGG/UFBA MESTRADO e DOUTORADO

\author{
Geofísica Aplicada \\ Geofísica Pura e Ambiental \\ Rua Barão de Geremoabo, s/n \\ CEP 40170-290 - Salvador . BA \\ Tel.: (071) 237-0407
}

E-mail: cpgg@cpgg.ufba.br - Internet: http:I|www.cpgg.ufpa.br 